\title{
Research Paper \\ Effect of Foreperiod Duration and Handedness on Simple and Choice Auditory Reaction Time Among the Older People
}

\author{
Somaye Rezaeimanesh ${ }^{1}$, ${ }^{*}$ Ebrahim Norouzi ${ }^{2}$, Sajad Parsaei ${ }^{1}$, Nahid Shetab Boushehri ${ }^{1}$, Rasoul Norouzi seyed hossieni ${ }^{3}$, Narciso \\ Gonzalez Vega ${ }^{4}$
}

1. Department of Sports Psychology, Faculty of Physical Education and Sport Sciences, Shahid Chamran University of Ahvaz, Ahvaz, Iran.

2. Department of Motor Behavior and Sport Management, School of Sport Sciences, Urmia University, Urmia, Iran.

3- Department of Physical Education, Faculty of Humanities, Tarbiat Modares University, Tehran, Iran.

4- Department of Social Sciences and Philosophy, Faculty of Humanities and Social Sciences, University of Jyväskylä, Jyväskylä, Finland.

\begin{tabular}{|c|c|}
\hline $\begin{array}{l}\text { Use yur device to scan } \\
\text { and read the article online }\end{array}$ & tation: Rezaeimanesh S, Norouzi E, Parsaei S, Shetab Boushehri N. [Effect of Foreperiod Duration and Handedness \\
\hline 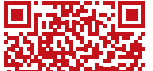 & $\begin{array}{l}\text { on Simple and Choice Auditory Reaction Time Among Elders (Persian)]. Iranian Journal of Ageing. 2017; 11(4):528-537. } \\
\text { http://dx.doi.org/10.21859/sija-1104538 }\end{array}$ \\
\hline 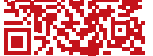 & doi): http://dx.doi.org/10.21859/sija-1104528 \\
\hline
\end{tabular}

Received: 18 May 2016 Accepted: 21 Sep. 2016

Key words:

Preparation, Handedness, Reaction

time, Aging

\section{ABSTRACT}

Objectives Cognitive functions such as reaction time undergo change with aging. Given the importance of preparation in response to reaction time in various life situations, this study aimed to examine the effect of various foreperiods $(0.5,1.5,2.5$, and $3.5 \mathrm{~s})$, and handedness at the time of simple and choice auditory reaction time.

Methods \& Materials The current research followed a quasi-experimental methodology and was carried out using a self-made software. The study population comprised all old people living in Ahvaz City. Of them, 30 people were selected through purposive sampling method and allocated into two groups of right- and left-handed people. Each individual was randomly tested for simple and selective reaction with randomly chosen foreperiods.

Results The findings demonstrated a significant difference $(P<0.05)$ between the different periods, and between simple and choice reaction time. It means that short (0.5 and $1.5 \mathrm{~s})$ foreperiods result in the increase of reaction time and that left-handed individuals are associated with shorter reaction times compared with their right-handed counterparts.

Conclusion Since cognitive processing occurs slower in the elderly, it is recommended that foreperiods with very short durations be avoided when preparing elders for different motor skills. In addition, the dominance of the right hemisphere, which contains higher numbers of neurons, among the left-handed might lead them to possess lower reaction times compared with right-handed individuals.

\section{* Corresponding Author:}

Ebrahim Norouzi, PhD Candidate

Address: Department of Motor Behavior and Sport Management, School of Sport Sciences, Urmia University, Urmia, Iran.

Tel: +98 (912) 9469087

E-mail: ebrahim.norouzi68@gmail.com 


\section{تأثير طول ييش}

سميه رضايىمنش'، "ابراهيم نوروزى'، سجاد بارسايى'، ناهيد شتاب بوشهرى' ، رسول نوروزى سيدحسينى"، نارسيسو كونزالس وكا"

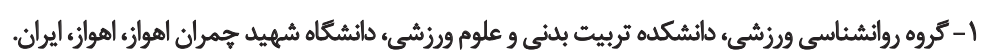

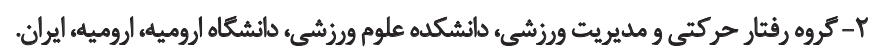

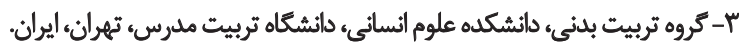

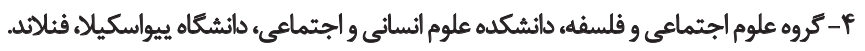

\section{באיט}

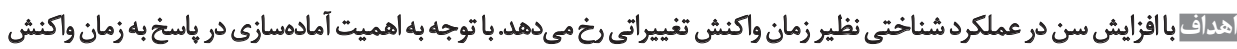

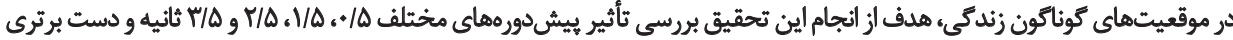

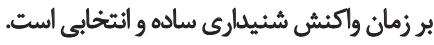

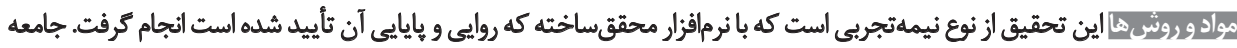

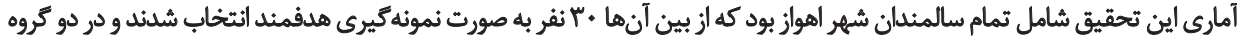

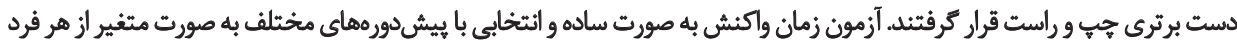

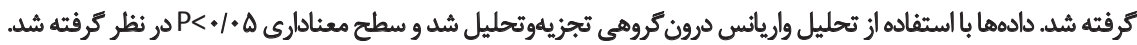

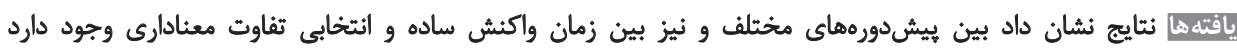

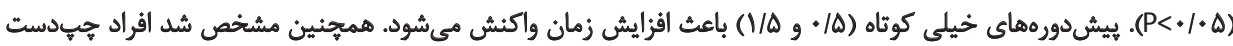

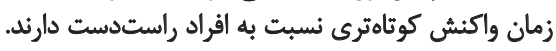

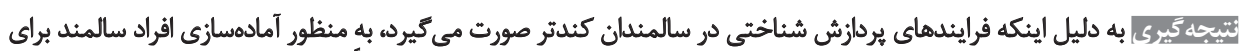

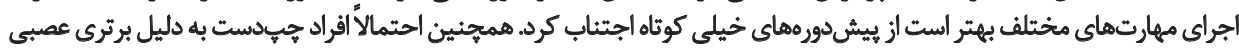

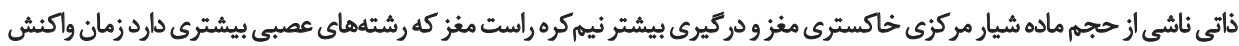
كوتاهترى نسبث به افراد راستدست ماديار مارند.

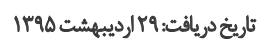
تاريخ بذيرش: آTهريور هوست

عوامل تخريبكننده فعاليت حركتى و شناختى و يافتن راههاى

مقدمه

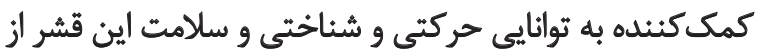

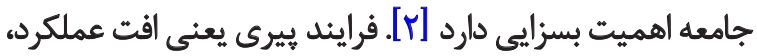

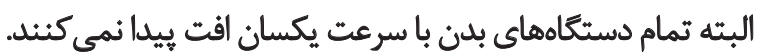

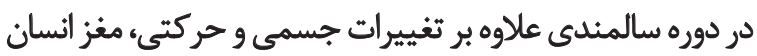

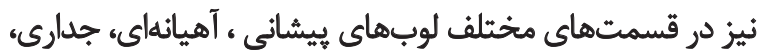

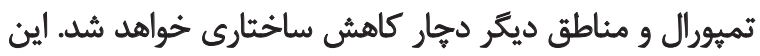

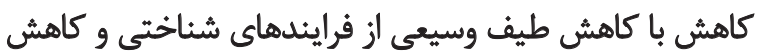

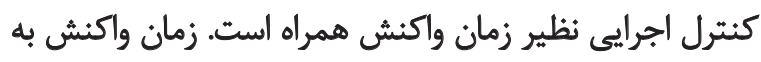

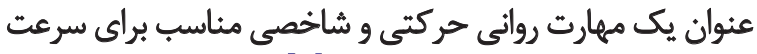

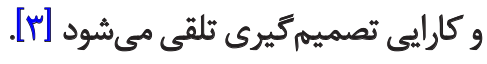

زمان واكنش (ساده و انتخابى و افتراقى) شاخص دقيقى براي

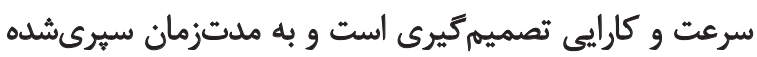

جيرشدن جمعيت و افرزايش جمعيت سالمندان به يك يدايده

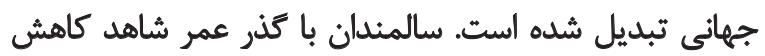

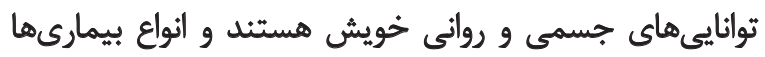

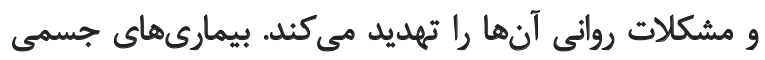

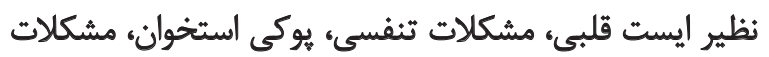

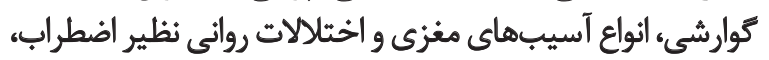

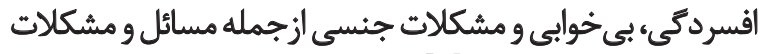

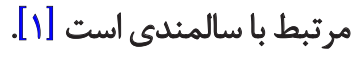

آمارهاى جهانى نشان مى دهد روزبهروز بر جمعيت سالمندان

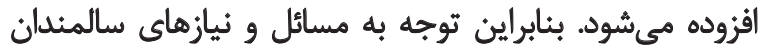

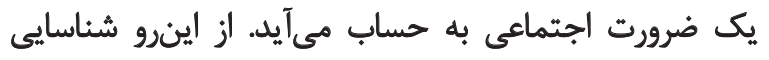


ادراكى حركتى به رويدادى است كه وقوع آن در آينده بيشيشينى زئي

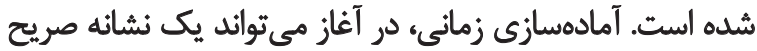

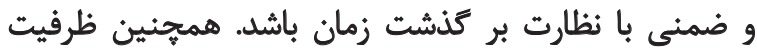

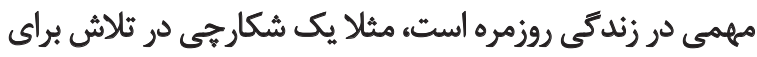

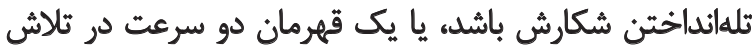

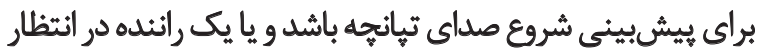

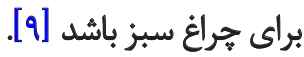

فاصله زمانى بين ارائه علامت آمادمباش تا ظطهور محرك اصلى بردي

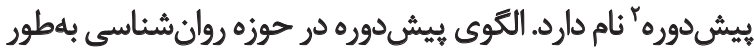

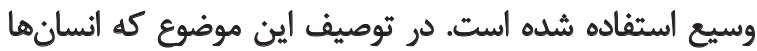

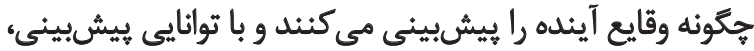

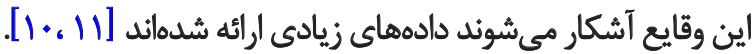

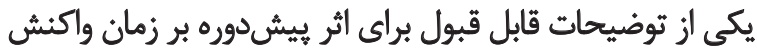

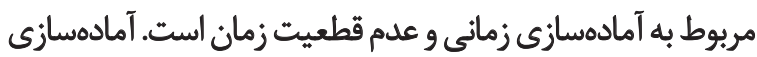

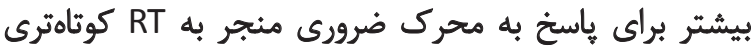

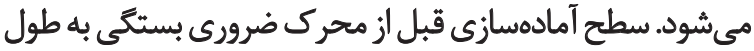

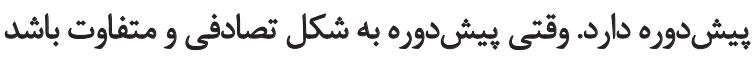

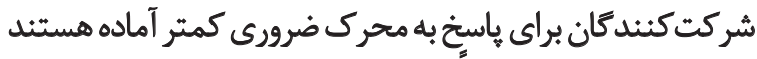

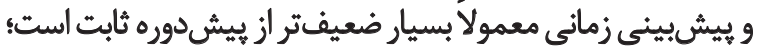

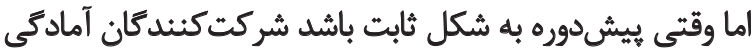

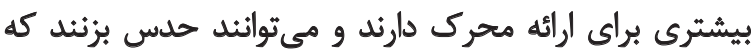

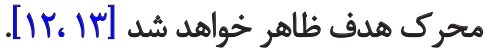

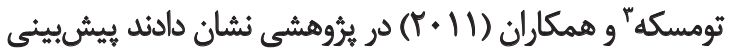

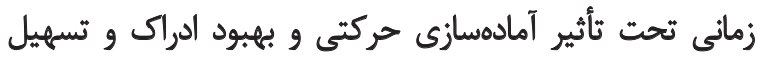

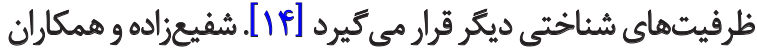

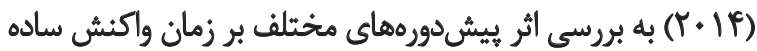

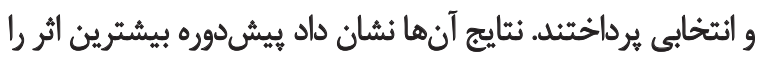

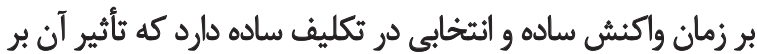

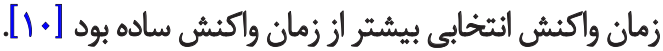

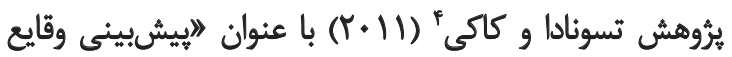

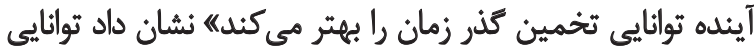

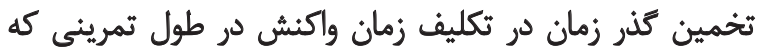

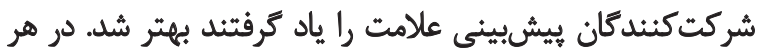

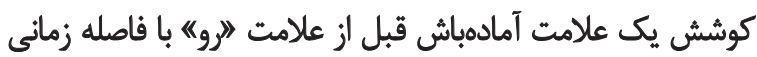

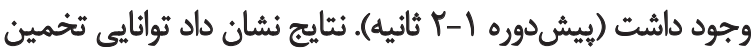

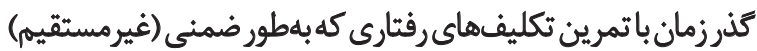

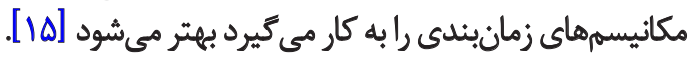
بدن انسان اندامهاى زوج بسيارى دارد كه از نظر ساختمانى و

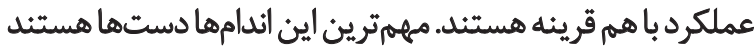

\section{Foreperiod}

3. Thomaschke

4. Tsunoda \& Kakei

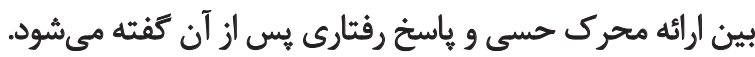

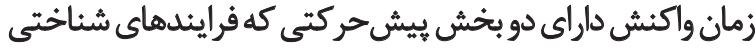

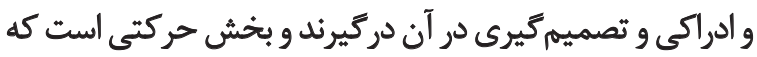

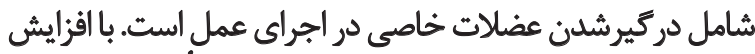

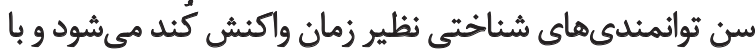

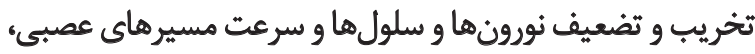

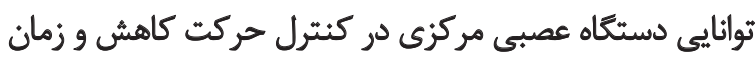

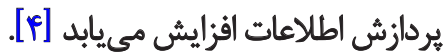

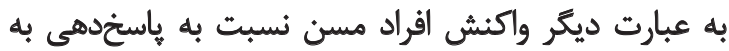

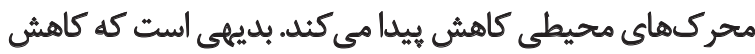

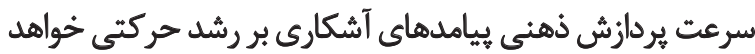

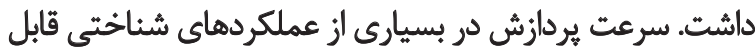

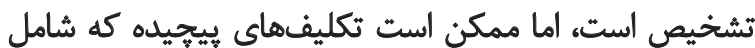

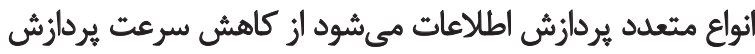

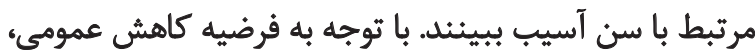

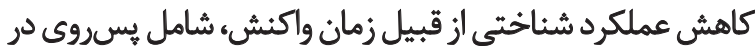

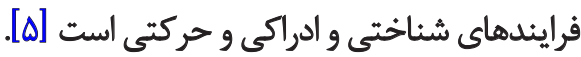

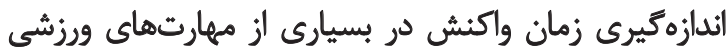

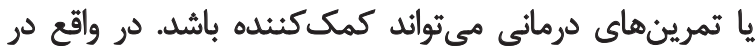

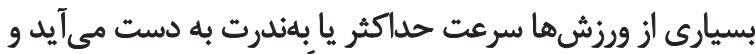

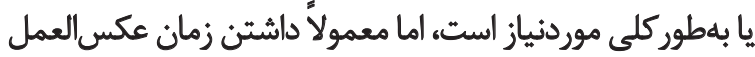

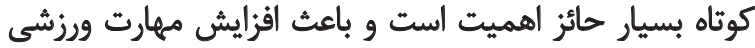

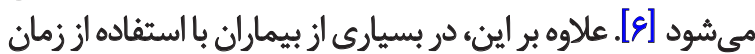

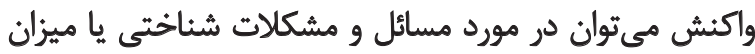

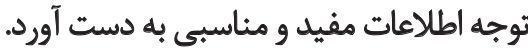

در محيط آزمايشكاه از اين متغير به عنوان يك روش قابل مشئ مشاهده

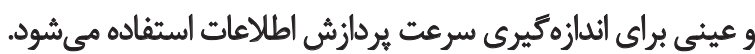

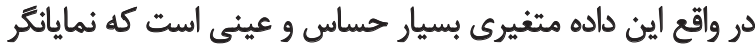

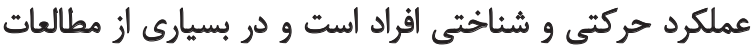

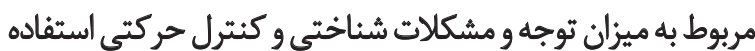

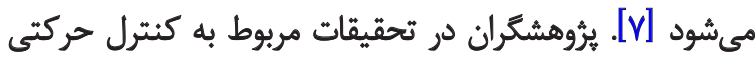

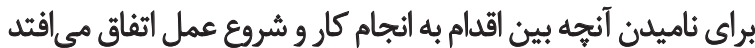

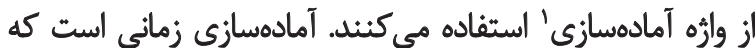

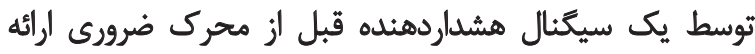

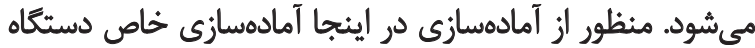
حركتى است كه فقط قبل از آغاز عمل فعال مى إشود [A]

الكوى بيشدوره ابزارى بالرزش براى دست كارى مقدار

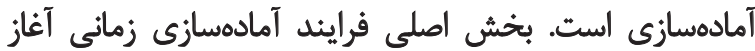

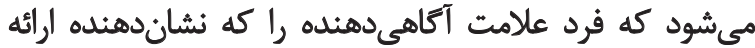

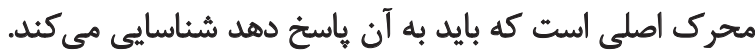
آمادهسازى زمائى نوعى توانايى از بيش فيث فعالشده توسط سيستم

1. Preparation 


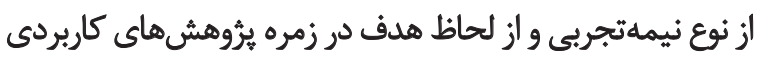

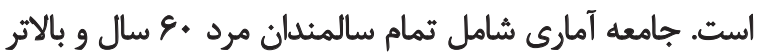

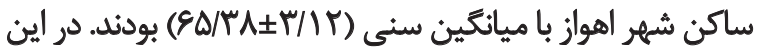

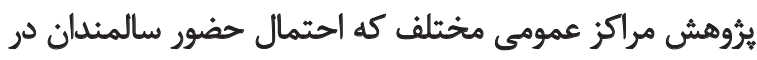

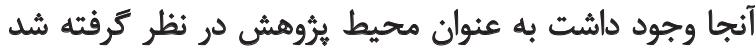

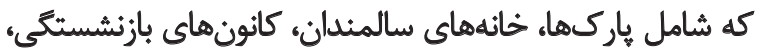

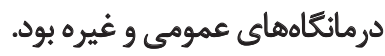

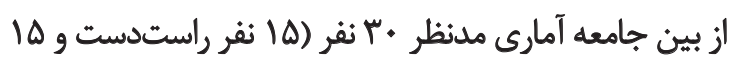

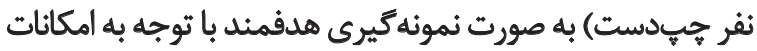

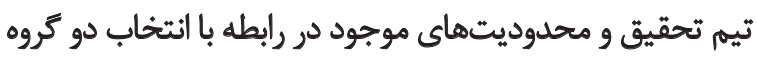

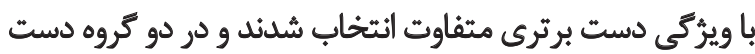

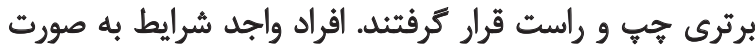
داوطلبائه در يُروهش شرك ركت كردند.

ازجمله ملاك هاى ورود به مطالعه مى توان به هوشيارى كامل،

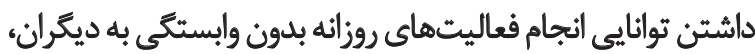

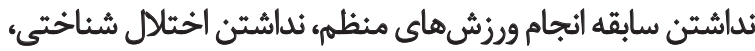

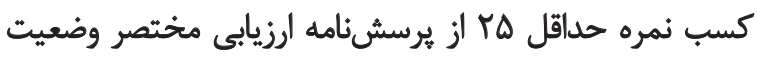

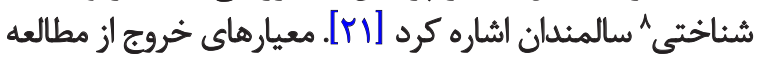

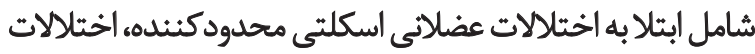

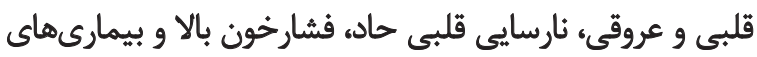

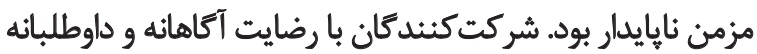

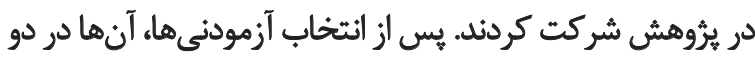

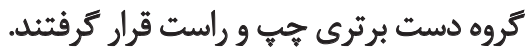
ابئار جمع آورى اطلاعات

\section{نرمافزار ييشدوره بر زمان واكنش ديدارى و شنيدارى}

محقق به كمك متخصصان نرمافزار اقدام به طراحى و ساخت

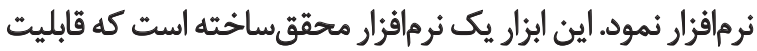

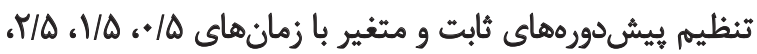

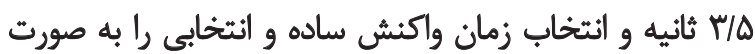

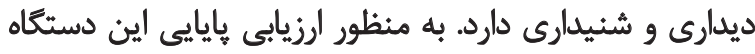

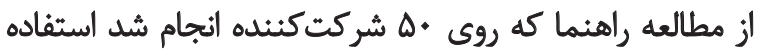

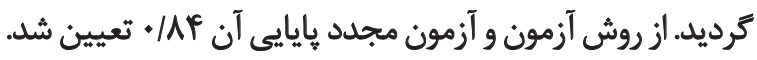

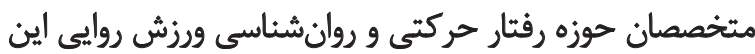

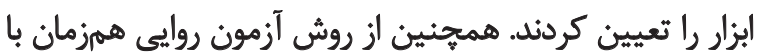

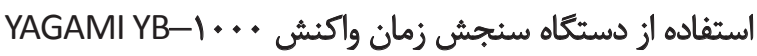

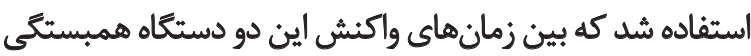

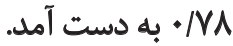

$$
\text { يرسش نامه ارزيابى مختصر وضعيت شناختى }
$$

8. Mini-Mental State Examination (MMSE)

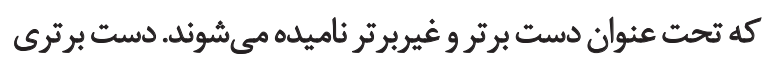

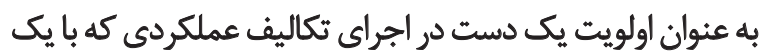

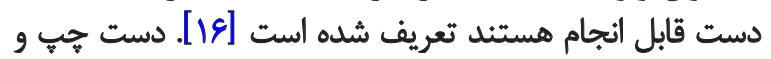

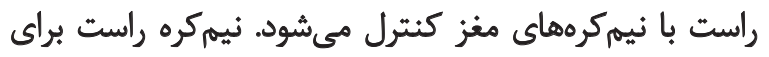

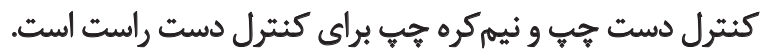

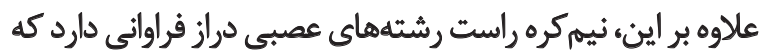

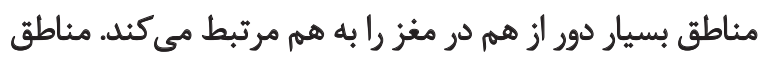

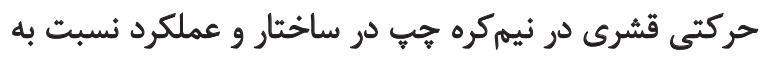

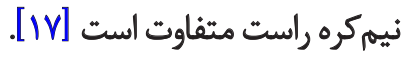

برخى از مطالعات كه به بررسى تثفاوت دست برتئى يرداختهاند

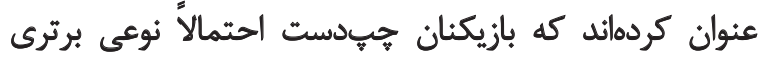

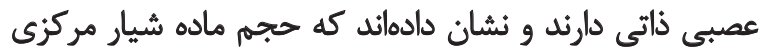

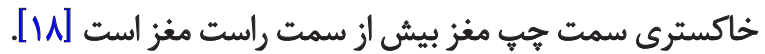

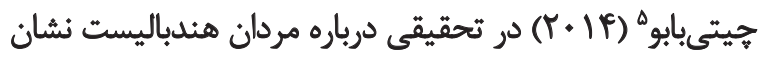

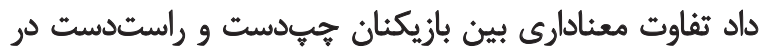

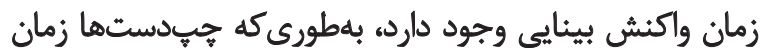
واكنش كوتاهترى دارند [19]

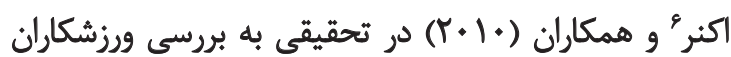

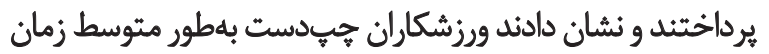

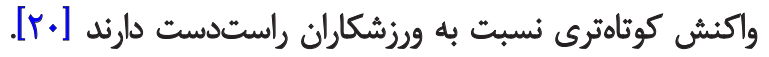

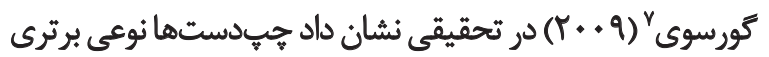

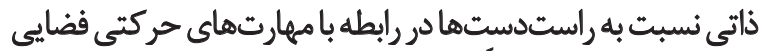

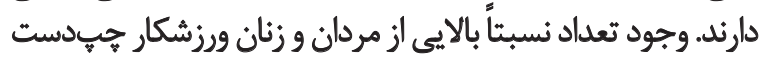

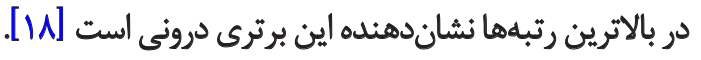

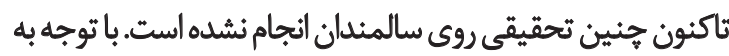

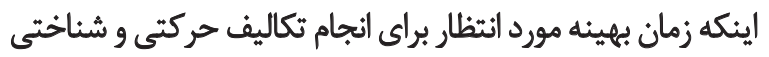

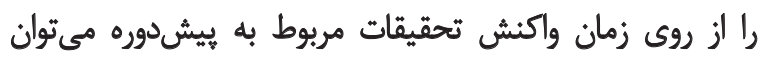

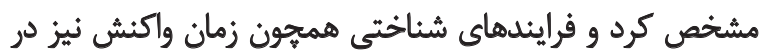

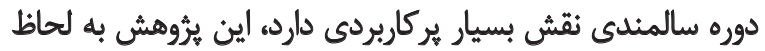

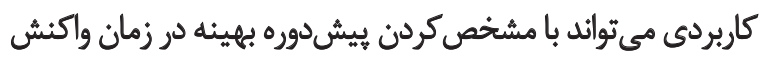

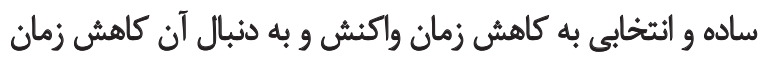

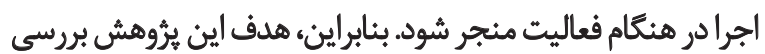

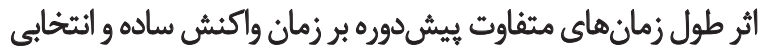
شنيدارى سالمندان در دو كروه با دست برتر راست و وجيً است.

$$
\text { קوش مطاله d }
$$

شرايطى از قبيل انكيزه، ميزان خواب آزمودنىها در شب قبل

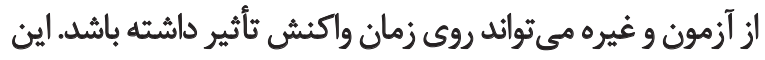

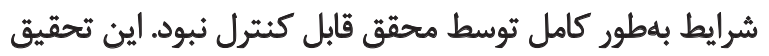

\section{Chittibabu \\ 6. Eckner \\ 7. Gursoy}




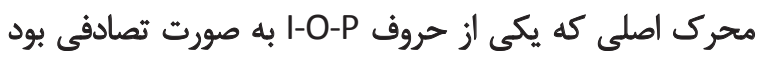

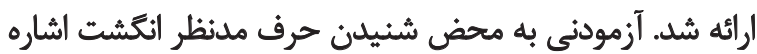

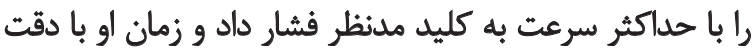

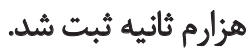

تجزيلوتحليل دادها

از ميانكين و انحرافمعيار به عنوان آمار توصيفى استفاده شد.

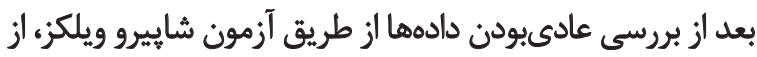

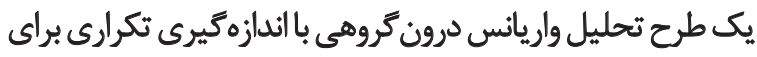

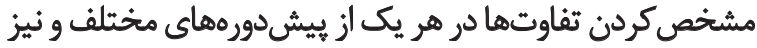

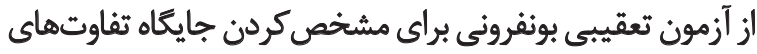

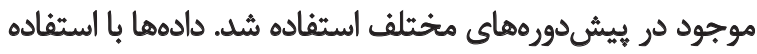

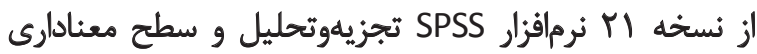

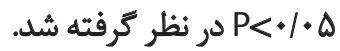

ياقتهها

ابتدا اطلاعات توصيفي دادهها درباره زمان واكنش ساده و

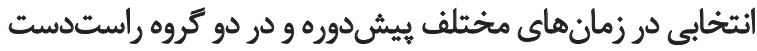

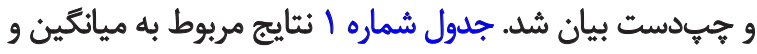

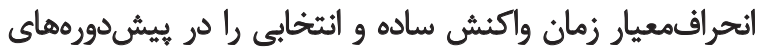

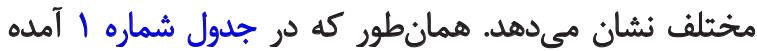

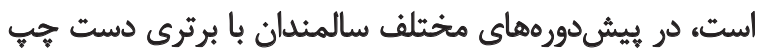
زمان واكنش شنيدارى ساده و انتخابى سريعترى دارئد.

به منظور بررسى زمان واكثش شنيدارى ساده و انتخابى در

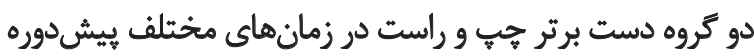

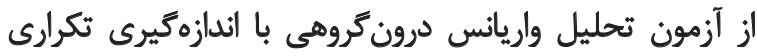

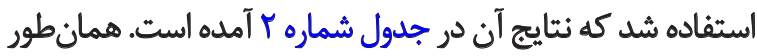

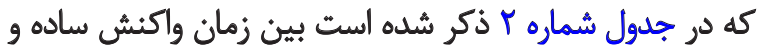

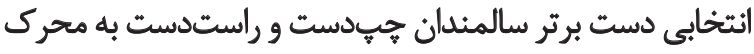

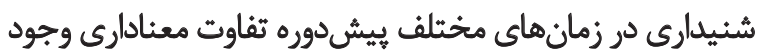

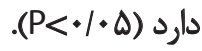

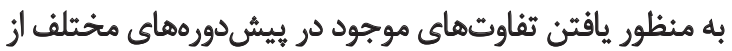

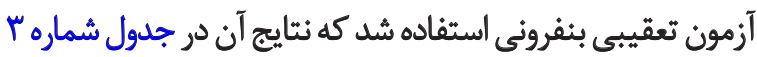

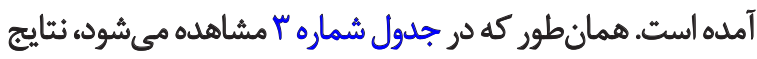

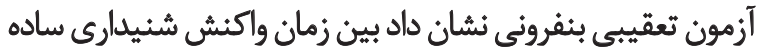

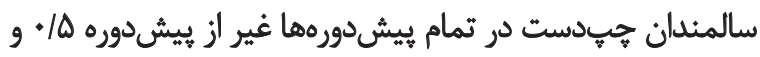

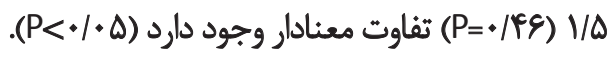

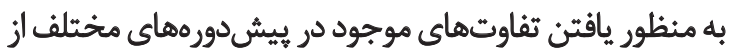

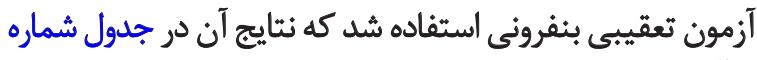

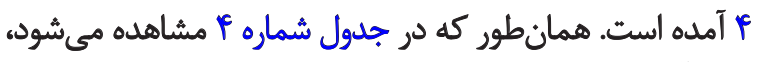

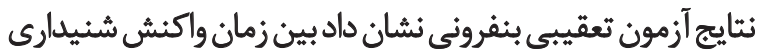

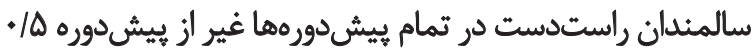

براى ارزيابانى وضعيت شناختى سالمندان از نسخه فارسى

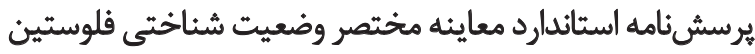

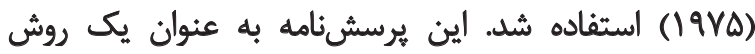

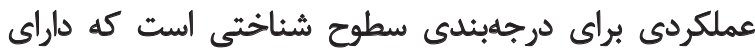

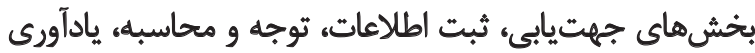

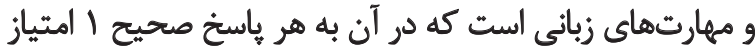

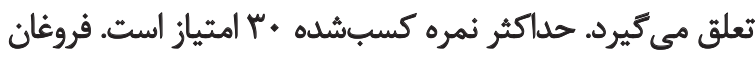

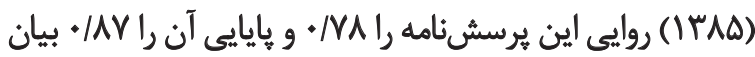

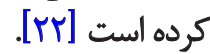

\section{روش الجراي تحقيث}

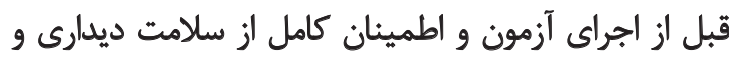

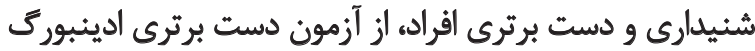

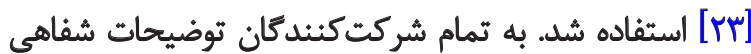

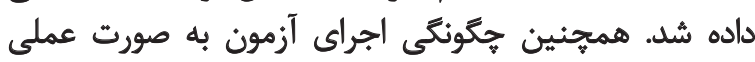

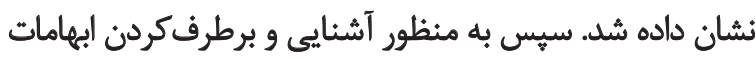

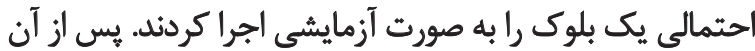
آزمون اصلى اجرا شد.

\section{آزمون ييشدوره بر زمان واكنش ساده شنيدارى در دست برتر}

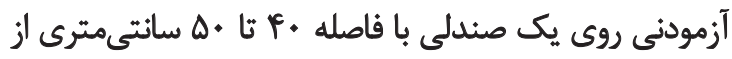

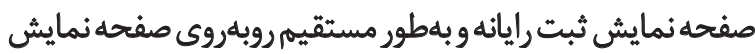

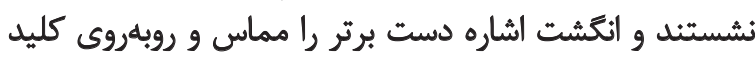

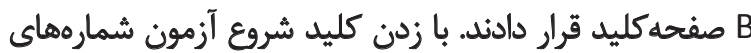

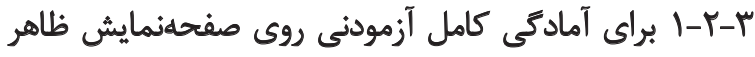

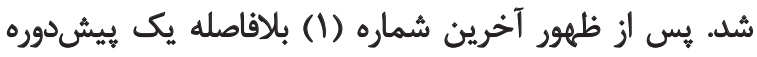

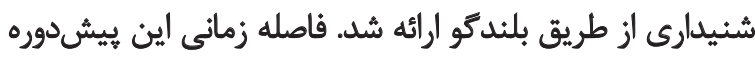

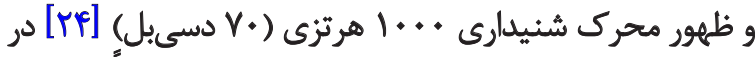

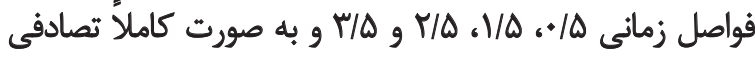

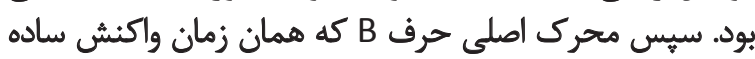

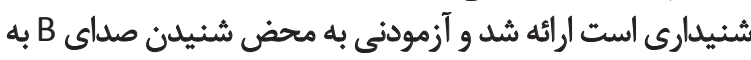

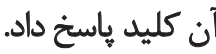

\section{آزمون بيش بردوه بر زمان واكنش انتخابى شنيدارى در دست برتر}

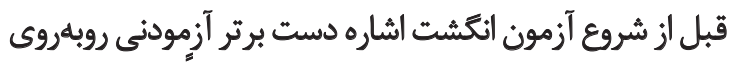

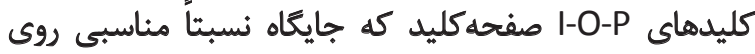

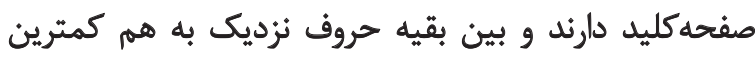

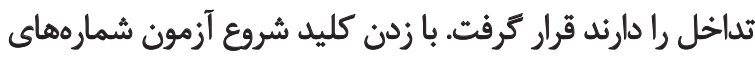

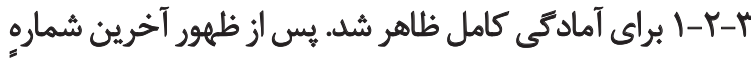

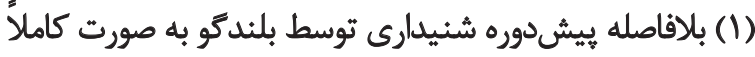

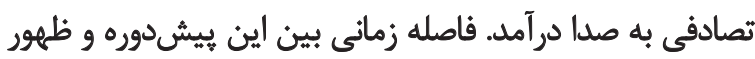

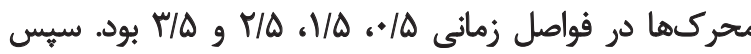


جدول ا. ميانكين و انحرافمعيار زمان واكنش شنيدارى ساده و انتخابى برحسب ثائيه در هيشدورهاي مختلف در افراد با دست برتر.

\begin{tabular}{|c|c|c|c|c|c|c|c|c|c|}
\hline \multicolumn{2}{|c|}{ بيش دوره ه/ ب ثائيه } & \multicolumn{2}{|c|}{ ييش دوره Y/ه ثانيه } & \multicolumn{2}{|c|}{ ييش دوره ه/ اثائيه } & \multicolumn{2}{|c|}{ ييش دوره ه/ + ثانيه } & \multirow{2}{*}{ دست } & \multirow{2}{*}{ متغير } \\
\hline انتحرافـمعيار, & مياتكين & انحرافمعيار & مياتكين & انحرافمعيار & مياتعين & أحرافمعيار & مياتكين & & \\
\hline.$/ 48$ &.$M I r$ &.$/ M Y$ & . $M T H$ & .111 & - Maf &.$/ 19 q$ & - /raq & راست & زمان واكثش \\
\hline.$/ N F$ & - TAA &.$/ 8$ & (ז9 & - ( & . Raq &.$/ P$ & . MTQ & جे & شنيدارى ساده \\
\hline.$/ p f$ & $\cdot / 4 \cdot r$ &.$/ 19$ & - /TRA & .11 & - Mte &.$/ . r$ & - Ifta & راست & زمان واكنش \\
\hline ( D &.$/ 419$ &.$/ M V$ & $.1 \Delta \cdot \varphi$ & .118 & . laqP &.$/ \pi$ & . /DFV & جي & شنيلاري انتخابي \\
\hline
\end{tabular}

는

جدول ب. تحليل واريانس درون كروهى با اندازهكيرى تكرارى در زمان واكثش شنيدارى ساده و انتخابى دست برتر سالمندان جيهدت و راستدست در زمانهاى بيش دوره مختلف.

\begin{tabular}{|c|c|c|c|c|c|c|c|c|}
\hline مجذور اتا & معنادارى سطح & $\mathbf{F}$ & ميانكين مجذورات & درجه آزادى & مجذورات مجموع & & آزمون & دست برتي \\
\hline \multirow{2}{*}{$.18 P$} & \multirow{2}{*}{$.1+\ldots+1$} & \multirow{2}{*}{$9 V / N 1$} & $8 N \mid \Delta / M^{P}$ & r & Y.PAS/TH & عامل & زمان واكنش & \multirow{4}{*}{ جي } \\
\hline & & & $e q / V A$ & $\Delta V$ & ravN.1 & خُطا & ساده & \\
\hline \multirow{2}{*}{.$/ 49$} & \multirow{2}{*}{$+1+\infty+1$} & \multirow{2}{*}{$18 / 10$} & I9TV/EV & r & DYAT/IT & 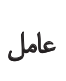 & زمان واكنش & \\
\hline & & & 119/r & $\Delta Y$ & $8 \lambda \cdot r / M$ & خططا & أتتخابى & \\
\hline \multirow{2}{*}{. } & \multirow{2}{*}{$+1+\infty 1$} & \multirow{2}{*}{$V / \Delta}$. & $189 . / 49$ & r & $r q 1 / m$ & عامل & زمان واكنش & \multirow{4}{*}{ راست } \\
\hline & & & TrV/r. & $\Delta V$ & $|K E+N|$ & خطا & ساده & \\
\hline \multirow{2}{*}{.$/ 2 A$} & \multirow{2}{*}{$.1+\infty+1$} & \multirow{2}{*}{ rq/p. } & riffer & $r$ & शPTT/8. & عامل & زمان واكتش & \\
\hline & & & $1.8 / 9 r$ & $\Delta V$ & $9.9 \% / q$. & خطا & خابي & \\
\hline
\end{tabular}

L

جدول rا. يافتههاى آزمون بنفرونى به منظور بررسى جايكاه تفاوت بيشدورهها در زمان واكنش شنيدارى سالمئدان با دست برتر جي.

\begin{tabular}{|c|c|c|c|c|}
\hline سطح معنادارى & خطاى استاندارد & تفاوت ميانكينها & (J) & كروه (I) \\
\hline .178 & $\mathrm{r} / \mathrm{MA}$ & -.1 .0 & 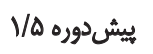 & \\
\hline $.1 . .9^{\circ}$ & $\mathrm{r} / \mathrm{\Delta A}$ &.$/ N$ & ييشدوره T/ه & ييش دوره 101. \\
\hline$+1+\cdots+$ & r/To &.$/ M$ & بيش دوره به/ه & \\
\hline.$/ . r^{*}$ & $r / r A$ &.$- / 1 F$ & Tيشدوره T/D & ييش دوره 1/ه \\
\hline $.1 .1^{\circ}$ & $r / \Delta 9$ &.$- / T A$ & ييشدوره M/Q M & \\
\hline$. / 4+$ & $F / \Delta F$ & $-+/ 11$ & ييش دوره & ييش دوره \\
\hline
\end{tabular}

هدف ثرؤهش حاضر بررسى تأثير ييش دور ههاى مختلف و دست

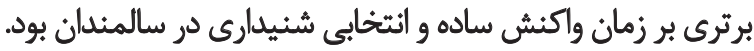

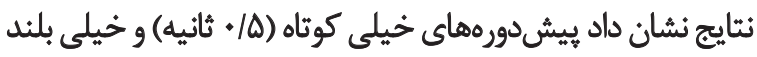

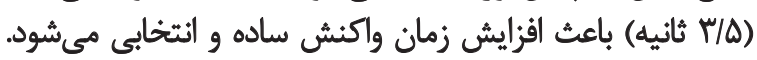

و

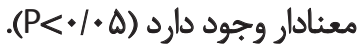




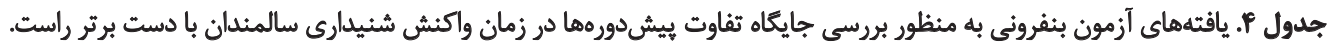

\begin{tabular}{|c|c|c|c|c|}
\hline سطح معنادارى & خطاى اسثائدارد & تفاوت مياتئينها & مروه (J) & مروه (I) \\
\hline$V *$ & r/gr & $* 4$ & ييش دوره V/ه & \multirow{3}{*}{ ايش دوره ها. } \\
\hline $.1+. .1^{*}$ & t/ere & .18 & Tيش موره ه/ه & \\
\hline .10 .4 & $r / T A$ &.$- / 11$ & ييش دوره r/ه & \\
\hline $.1 . .1 *$ & r/ge & $-\cdot / 1$ & تيشدوره & \multirow[b]{2}{*}{ ييش دوره ه/ه } \\
\hline $.1 \cdot .1^{*}$ & $t / R P$ &.$- / 19$ & ييشدوره ه/ه & \\
\hline . / Fr & $r / \Delta F$ & $-\cdot / \pi r$ & ييشدوره باه & يسش دوره ه/ه \\
\hline
\end{tabular}

L

آنها نشان داد در شرايط آمادهباش، ييشدوره كوتادياهتر از حالت

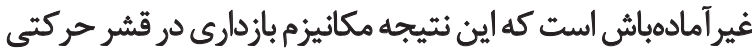

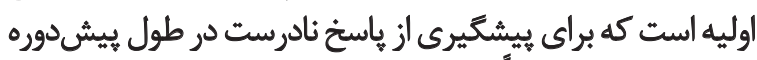

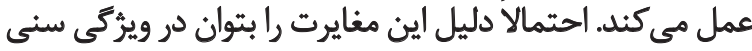

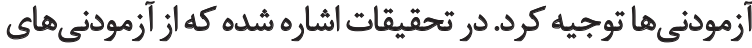

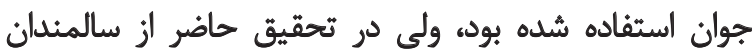
استفاده شد. تحقيقات مختلفى نشان دادهاند فرايندهاى شئى شناختى

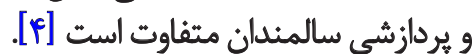
دليل ديكر ناهمخوانيى را مى توان در نحوه ظهور محرك دها دانست.

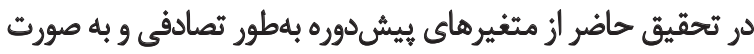

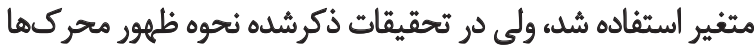

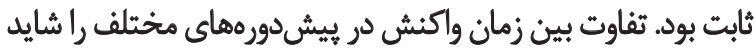

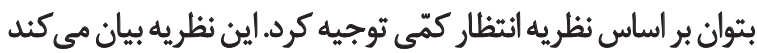

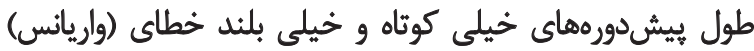

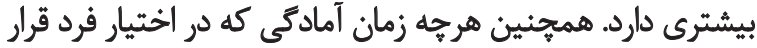

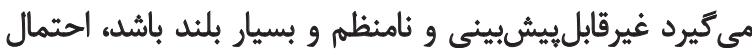

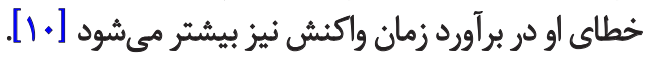
در ارتباط با بيشدوره و زمان واكنش دو ديدكاه متفاوت وجود

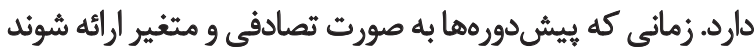

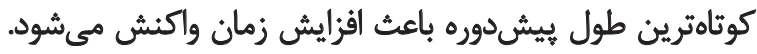

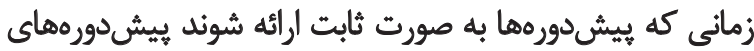

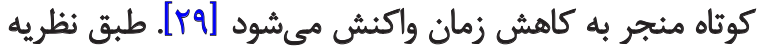

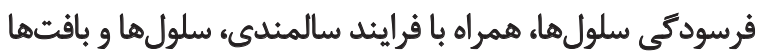

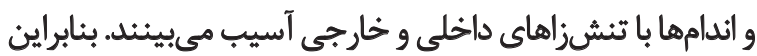

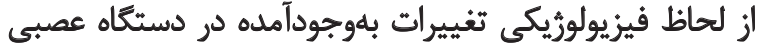

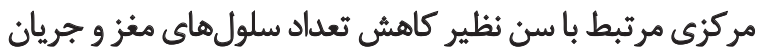

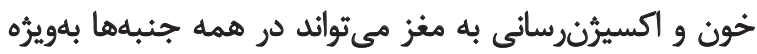

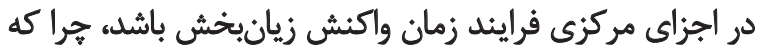

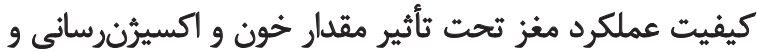

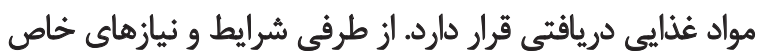
يك تكليف، سرعت عكس العمل و تصميم كيرى رادر إفر افراد سالمند

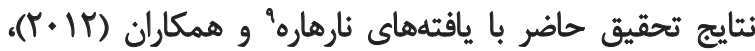

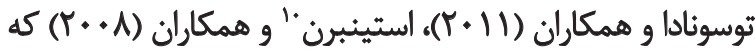

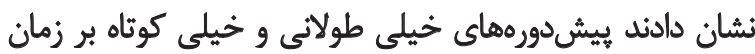

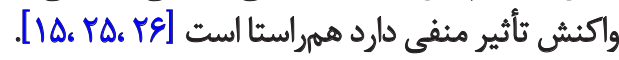
يبيش دوره خيلى كوتاه زمان خيلى كمى را براي آمادهكردن فرددر

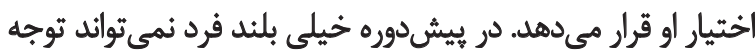

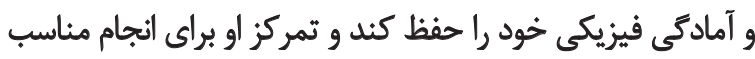

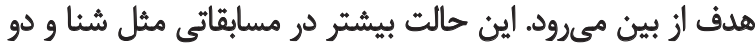
سرعت كه شروع آن بسيار مهم است ميت متواند كاربرد داشته باشد.

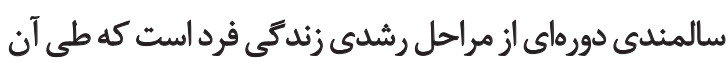

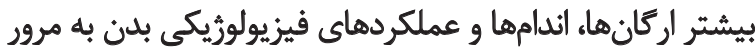

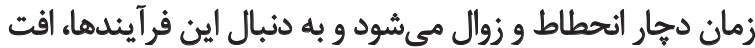

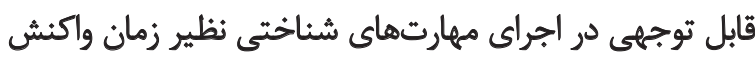

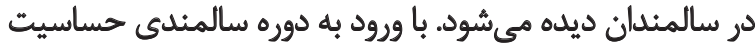

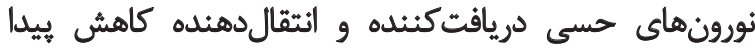

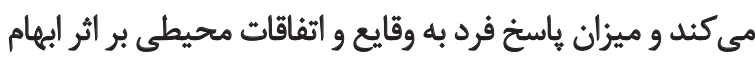

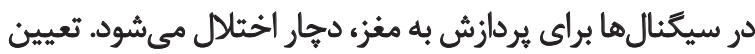

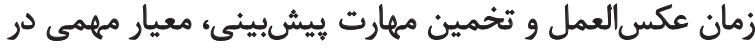

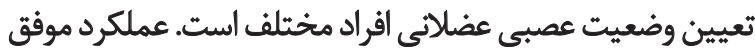

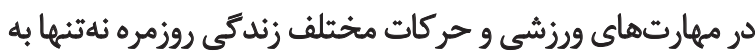

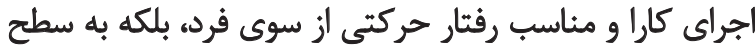
بالايى از توانايى ادراكى او نياز دارد [TrV]

نتايج اين تحقيق با يافتهاي شفيعزاده و همكاران (T) (T)

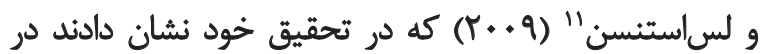

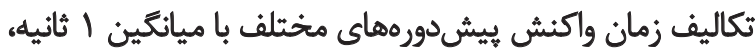

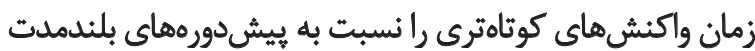

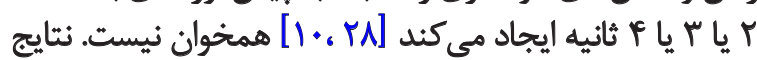

9. Narhare

10. Steinborn

11. Leth-Steensen 


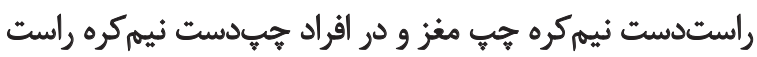

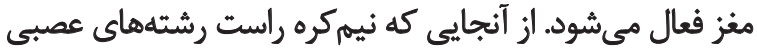

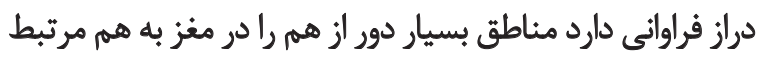

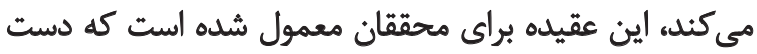

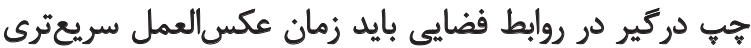

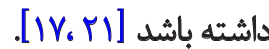

\section{نتيجه كيرى نهايی}

به دليل اينكه فرايندهاى يُردازش شناختى در سالمندان كندتر

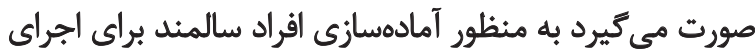

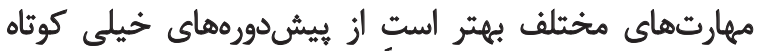

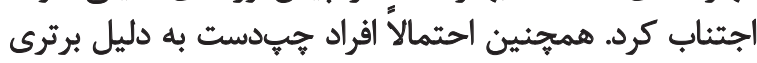

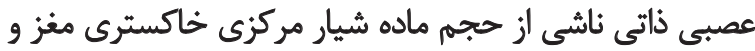

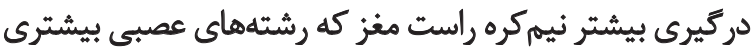

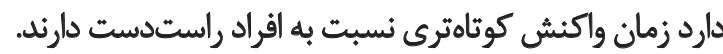

$$
\text { تشكر وقدردانى }
$$

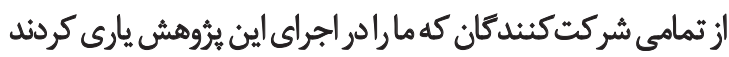

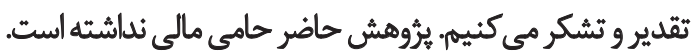

بهبود مي بخشد. [.r.]

نتايج نشان داد زمان واكنش انتخابى نسبت به زمان واكنش

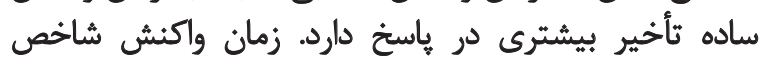

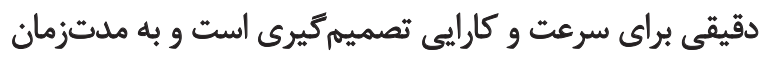

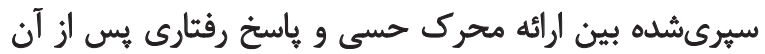

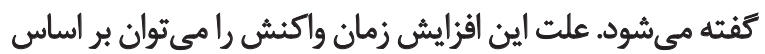

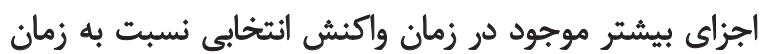

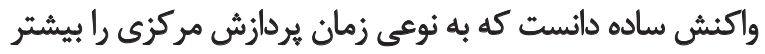

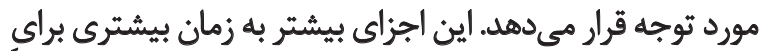

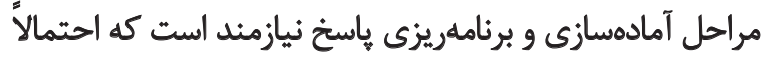

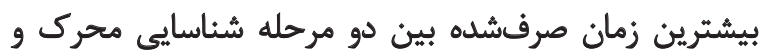

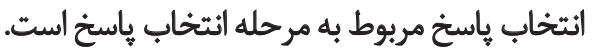

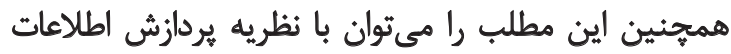

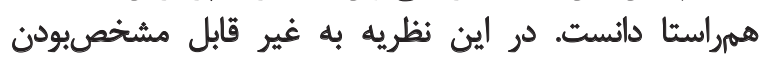

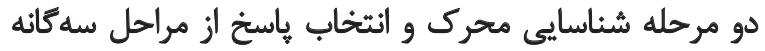
بردازش اطلاعات زمان واكنش انتخابى نسبت به زمان زمان وان واكنش

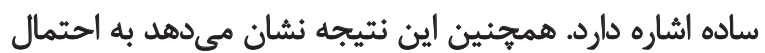

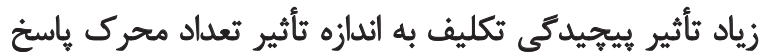

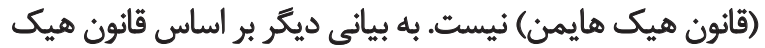

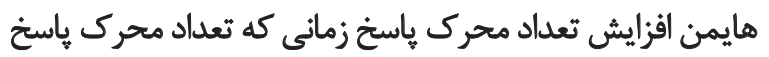

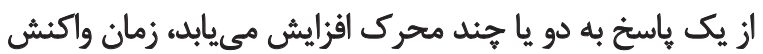
نيز بيشترين افزايش را دارد [ـ[اسئ.

نتايج ديكر اين يُؤهش نشان داد افراد بادست برترى جب زبت زمان

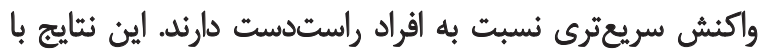

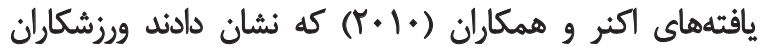

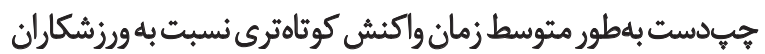

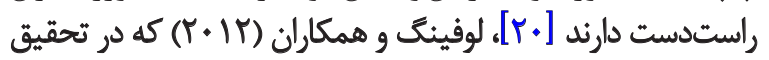

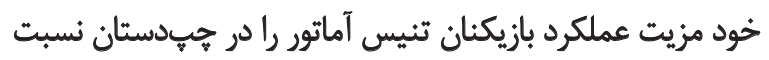

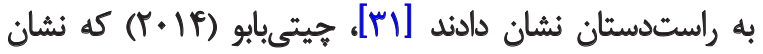

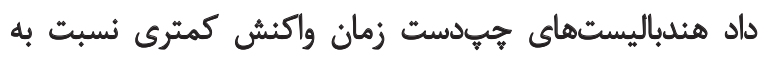

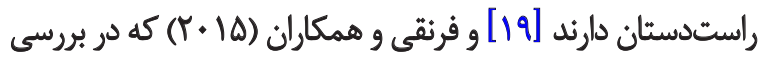

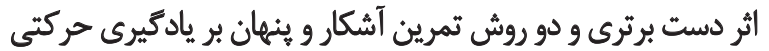

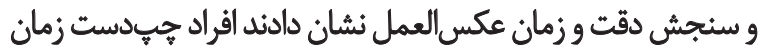
واكنش كمترى دارند [بr] همخوانى دارد.

در تكاليفى كه با دست راست انجام مىشود قشر حركتى اوليه

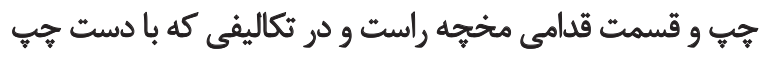

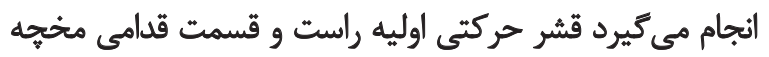

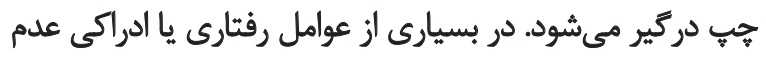

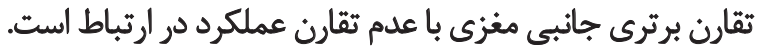

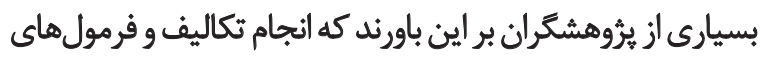

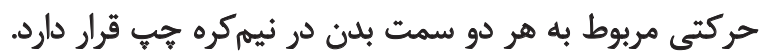
محققان دريافتند در اجراى تكاليف حركتى با دست برتئ در در در افراد 


\section{References}

[1] Marengoni A, Angleman S, Melis R, Mangialasche F, Karp A, Garmen A, et al. Aging with multimorbidity: A systematic review of the literature. Ageing Research Reviews. 2011; 10(4):430-9. doi: 10.1016/j.arr.2011.03.003

[2] Karimi Torghabeh E, Ehsani M, Koozechian H, Mehrabi Y. جEffect of 16 weeks walking with different dosages on psychosocial function related quality of life among 60 to 75 years old men (Persian)]. Iranian Journal of Ageing. 2011; 5(18):21-29.

[3] Rahmani M, Heirani A, Yazdanbakhsh K. [The effect of pilates training on improving the reaction time and balance of sedentary elderly men (Persian)]. Journal of Modern Rehabilitation. 2014; 9(3):44-53.

[4] Payne G, Isacs L. Human motor development: A lifespan approach. $5^{\text {th }}$ ed. Philadelphia: McGraw Hill Pub; 2002.

[5] Kahrizi N. [The effect of Uoga's training on improving the reaction time, balance static and dynamic of sedentary elderly men in Kermanshah (Persian)] [MSc. thesis]. Kermanshah: University of Kermanshah; 2013.

[6] Ellemberg D, St-Louis-Deschênes M. The effect of acute physical exercise on cognitive function during development. Psychology of Sport and Exercise. 2010; 11(2):122-6. doi: 10.1016/j.psychsport.2009.09.006

[7] Turhanoğlu AD, Beyazova M. Reaction time and movement time in patients with carpal tunnel syndrome: An electromyographic study. Clinical Biomechanics. 2003; 18(5):380-4. doi: 10.1016/s0268-0033(03)00053-6

[8] Magill Richard. A. Motor Control and learning concept and application. $9^{\text {th }}$ ed. Philadelphia: McGraw Hill Pub; 2011.

[9] Vallesi A, Lozano VN, Correa Á. Dissociating temporal preparation processes as a function of the inter-trial interval duration. Cognition. 2013; 127(1):22-30. doi: 10.1016/j.cognition.2012.11.011

[10] Shafizadeh A, Farokhi A, Namazizadeh M, Sheikh M. [Effect of foreperiod duration on simple and choice reaction time in simple and complex task (Persian)]. Motor Behavior. 2014; 6(16):121-138.

[11] MUller-Gethmann H, Ulrich R, Rinkenauer G. Locus of the effect of temporal preparation: Evidence from the lateralized readiness potential. Psychophysiology. 2003; 40(4):597-611. doi: $10.1111 / 1469-8986.00061$

[12] Laflamme V, Zakay D, Gamache P-L, Grondin S. Foreperiod and range effects on time interval categorization. Attention, Perception, \& Psychophysics. 2015; 77(5):1507-14. doi: 10.3758/ s13414-015-0937-y

[13] Cardoso-Leite P, Mamassian P, Gorea A. Comparison of perceptual and motor latencies via anticipatory and reactive response times. Perception \& Psychophysics. 2009; 71(1):82-94. doi: 10.3758/app.71.1.82

[14] Thomaschke R, Kiesel A, Hoffmann J. Response specific temporal expectancy: Evidence from a variable foreperiod paradigm. Attention, Perception, \& Psychophysic. 2011; 73(7):2309-22. doi: 10.3758/s13414-011-0179-6

[15] Tsunoda Y, Kakei S. Anticipation of future events improves the ability to estimate elapsed time. Experimental Brain Research. 2011; 214(3):323-34. doi: 10.1007/s00221-011-2821-x
[16] Van der Hoorn A, Burger H, Leenders KL, de Jong BM. Handedness correlates with the dominant Parkinson side: A systematic review and meta-analysis. Movement Disorders. 2011; 27(2):20610. doi: $10.1002 / \mathrm{mds} .24007$

[17] Nisiyama M, Ribeiro-do-Valle LE. Relative performance of the two hands in simple and choice reaction time tasks. Brazilian Journal of Medical and Biological Research. 2014; 47(1):80-9. doi: 10.1590/1414-431×20132932

[18] Gursoy R. Effects of left- or right-hand preference on the success of boxers in Turkey. British Journal of Sports Medicine. 2008; 43(2):142-4. doi: 10.1136/bjsm.2007.043547

[19] Chittibabu B. Comparison of repeated sprint ability and fatigue index among male handball players with respect to different playing position. International Journal of Physical Education Fitness and Sports. 2014; 3(1):71-5.

[20] Eckner JT, Kutcher JS, Richardson JK. Pilot evaluation of a novel clinical test of reaction time in national collegiate athletic association division i football players. Journal of Athletic Training. 2010; 45(4):327-32. doi: 10.4085/1062-6050-45.4.327

[21] Folstein MF, Folstein SE, McHugh PR. Mini-mental state: A practical method for grading the cognitive state of patients for the clinician. Journal of Psychiatric Research. 1975; 12(3):189-98. PMID: 1202204

[22] Foroughan M, Jafari Z, Shirinbayani P, Qaem Maqam Farahani Z. [Normalization of the brief physical examination of the cognitive status in older in Tehran (Persian)]. Advances in Cognitive Science. 2008; 10(2):29-37.

[23] Oldfield RC. The assessment and analysis of handedness: The Edinburgh inventory. Neuropsychologia. 1971; 9(1):97-113. doi: 10.1016/0028-3932(71)90067-4

[24] Steinborn MB, Rolke B, Bratzke D, Ulrich R. Dynamic adjustment of temporal preparation: Shifting warning signal modality attenuates the sequential foreperiod effect. Acta Psychologica. 2009; 132(1):40-7. doi: 10.1016/j.actpsy.2009.06.002

[25] Narhare P., B. Chaitra B, Vijay M. A comparative study of choice reaction time in young males and females. National Journal of Integrated Research in Medicine. 2012; 3(5):84-88.

[26] Steinborn MB, Rolke B, Bratzke D, Ulrich R. Sequential effects within a short foreperiod context: Evidence for the conditioning account of temporal preparation. Acta Psychologica. 2008; 129(2):297-307. doi: 10.1016/j.actpsy.2008.08.005

[27] Williams AM, Grant A. Training perceptual skill in sport. International Journal of Sport Psychology. 1999; 30(2):194-220.

[28] Leth-Steensen C. Lengthening fixed preparatory foreperiod durations within a digit magnitude classification task serves mainly to shift distributions of response times upwards. Acta Psychologica. 2009; 130(1):72-80. doi: 10.1016/j.actpsy.2008.10.003

[29] Näätänen R. The diminishing time-uncertainty with the lapse of time after the warning signal in reaction-time experiments with varying fore-periods. Acta Psychologica. 1970; 34:399-419. doi: 10.1016/0001-6918(70)90035-1

[30] Rahmani M, Heirani A, Yazdanbaksh K. [The effect of Pilates training on improving the reaction time and balance of sedentary elderly men (Persian)]. Journal of Development \& Motor Learning. 2014; 6(3):347-363. 
[31] Loffing F, Schorer J, Hagemann N, Baker J. On the advantage of being left-handed in volleyball: Further evidence of the specificity of skilled visual perception. Attention, Perception, \& Psychophysics. 2011; 74(2):446-53. doi: 10.3758/s13414-011-0252-1

[32] Fernaghi Z, Badami R, Nezakathostni M. [Effect of handedness and the type of training (overt and covert) on the accuracy and reaction time in a row (Persian)]. Journal of Development \& Motor Learning. 2015; 7(4):529-548. 